\title{
Optimal $\lambda$ based Economic Emission Dispatch using Simulated Annealing
}

\author{
Sasikala. J \\ Lecturer in Computer Science and \\ Engineering, \\ Annamalai University, Annamalainagar \\ 608 002, India.
}

\author{
Ramaswamy. M \\ Professor of Electrical Engineering, \\ Annamalai University, Annamalainagar \\ 608 002, India.
}

\begin{abstract}
The economic emission dispatch (EED) assumes a lot of significance to meet the clean energy requirements of the society, while at the same time minimising the cost of generation. The solution schemes in an attempt to arrive at the global best through the use of evolutionary algorithms are however inadequate to cater to problems of large size. The search based EED approaches are computationally inefficient particularly for problems with large number of
\end{abstract}

decision variables. This paper attempts to develop a new SA based modified approach with a single decision variable to solve the EED problem. The philosophy involves the introduction of a new decision variable through a prudent mathematical transformation of the relation between the decision variable and the optimal generations. It thus yields a reduction in the number of problem variables and contributes to realistically enhance the performance of the existing heuristic strategies. The feasibility of the proposed approach is evaluated through two test systems and the results are compared with the available methods to highlight its suitability for online applications.

\author{
Nomenclature \\ COST \\ ELD \\ EED \\ PA \\ SA \\ ESA \\ $a_{i} b_{i} \& c_{i}$ \\ $d_{i} e_{i} \& f_{i}$ \\ $I C_{i}$ \\ $I C_{i}^{\min } \& I C_{i}^{\max }$ \\ cost function \\ economic load dispatch \\ economic emission dispatch \\ proposed algorithm \\ simulated annealing \\ existing SA based ELD \\ fuel cost coefficients of $i^{\text {th }}$ generating plant \\ emission coefficients of $i^{\text {th }}$ generating plant \\ incremental cost at $i^{\text {th }}$ generation plant \\ minimum and maximum values of $I C_{i}$ respectively
}




$\begin{array}{ll}n g & \text { number of generating plants } \\ P_{D} & \text { total power demand } \\ P_{G i} & \text { generation at } i^{\text {th }} \text { generating plant } \\ P_{G i}^{\min } \& P_{G i}^{\max } & \text { minimum and maximum of } P_{G i} \text { respectively } \\ F_{i}\left(P_{G i}\right) & \text { fuel cost function of } i^{\text {th }} \text { generating plant in } \$ / h \\ E_{i}\left(P_{G i}\right) & \text { emission cost function of } i^{\text {th }} \text { generating plant in } \mathrm{kg} / \mathrm{h} \\ h_{i} & \text { price penalty factor of } i^{\text {th }} \text { generating plant in } \$ / \mathrm{kg} \\ T_{t} & \text { current temperature } \\ T_{t+1} & \text { next temperature } \\ \lambda & \text { incremental cost of received power } \\ \lambda^{\text {min }} \& \lambda^{\max } & \text { minimum and maximum values of } \lambda \text { respectively } \\ \Phi & \text { objective function to be minimized } \\ \Phi_{T} & \text { augmented objective function to be minimized } \\ \alpha & \text { cooling coefficient } \\ P(T) & \text { transition probability in the interval }[0,1] \\ \Delta F & \text { reduction in cost of the trial solution compared with the current }\end{array}$

\subsection{INTRODUCTION}

Economic Load Dispatch (ELD) plays an important role in maintaining a high degree of economy and reliability in power system operational planning. It is a computational process of allocating the total required generation among the available generating units subject to load and operational constraints such that the cost of operation is minimum. Various techniques such as lambda iteration, gradient search, linear programming, dynamic programming and Lagrangian relaxation are used to solve ELD problem [1-2]. Recently intelligent algorithms such as Pattern Search [3], Neural Networks [4-5], Genetic Algorithm [6], Simulated Annealing (SA) [7], Evolutionary Programming [8] and Particle Swarm optimisation [9] are applied to solve ELD problems.

Operating at absolute minimum cost can no longer be the only criterion for dispatching electric power due to increasing concern over the environmental considerations. The generation of electricity from fossil fuel releases several contaminants, such as sulphur dioxides, nitrogen oxides and carbon dioxide into the atmosphere. The pressing public demand for clean air and the enforcement of environmental regulations in recent years have changed the dispatch problem with conflicting objectives of minimising both the fuel cost and the emissions.

Several methods have been suggested for solving the multiobjective economic emission dispatch (EED) problem. A direct NR method based on alternative jacobian matrix [10], a recursive approach based on dynamic programming [11], a simplified recursive approach [12], a progressive articulation of preference information based optimisation technique [13] and an analytical strategy based on mathematical modelling [14] have been presented to handle combined EED problems. In recent years, heuristic optimisation techniques have aroused greater 
interest due to their flexibility, versatility and robustness. These evolutionary approaches such as an interactive fuzzy satisfying based SA technique [15], particle swarm optimisation based goal-attainment method [16], a multiobjective genetic algorithm [17] and a fuzzified multi-objective particle swarm optimisation algorithm [18] have been extensively articulated to obtain the global optimal solution. However, on account of the fact that EED problems necessarily involve a large number of problem variables, the heuristic approaches have been found to suffer from huge computational burden and end up with consuming exhaustively large execution times. Therefore an efficient strategy that is independent of the number of generating plants in the system, is formulated with a single decision variable and invokes the use of SA to solve for EED problem in this paper

\subsection{Problem Formulation}

The aim of EED is to minimise the total generation cost and emissions of a power system for a given load while satisfying various constraints [1-2]. The objective function is thus obtained by blending the emission cost function with the fuel cost function through the use of a price penalty factor [19] and the constrained optimisation problem is formulated as

$$
\text { Minimise } \Phi=\sum_{i=1}^{n g}\left\{F_{i}\left(P_{G i}\right)+h_{i} E_{i}\left(P_{G i}\right)\right\}
$$

Subject to

$$
\begin{aligned}
& \sum_{i=1}^{n g} P_{G i}-P_{D}=0 \\
& P_{G i}^{\min } \leq P_{G i} \leq P_{G i}^{\max } \\
& i=1,2, \cdots, n g
\end{aligned}
$$

where

$$
\begin{aligned}
& F_{i}\left(P_{G i}\right)=a_{i} P_{G i}{ }^{2}+b_{i} P_{G i}+c_{i} \\
& E_{i}\left(P_{G i}\right)=d_{i} P_{G i}{ }^{2}+e_{i} P_{G i}+f_{i} \\
& h_{i}=F_{i}\left(P_{G i}^{\max }\right) / E_{i}\left(P_{G i}^{\max }\right)
\end{aligned}
$$

\subsection{Classical $\lambda$ iteration method [1]}

The augmented lagrangian function for the ELD problem can be written as

$$
\text { Minimise } \Phi_{T}=\sum_{i=1}^{n g}\left\{F_{i}\left(P_{G i}\right)+h_{i} E_{i}\left(P_{G i}\right)\right\}-\left[\sum_{i=1}^{n g} P_{G i}-P_{D}\right]
$$

The co-ordination equation from the above function can be obtained as

$$
\frac{d F_{i}\left(P_{G i}\right)}{d P_{G i}}=2\left[a_{i}+h_{i} d_{i}\right] P_{G i}+\left[b_{i}+h_{i} e_{i}\right]=\lambda
$$

The above equation can be solved iteratively for EED imposing on itself the generator power limits and the power balance equation as constraints. This classical lambda-iterative technique is in use for a long time as it is simple and easy for online implementation. 


\subsection{Simulated Annealing}

The SA algorithm, proposed by Kirkpatrick et al in 1983 [20], is a powerful optimisation technique, which exploits the resemblance between a minimisation process and the annealing process of the molten metal. The annealing process begins with a high temperature and the metal is slowly cooled so that the system maintains the thermal equilibrium at every stage, until the energy of the system acquires the global minimum value. The physical annealing process is simulated in the SA technique for the determination of global or near-global optimal solutions of the difficult combinatorial optimisation problems involving non-linear objective functions and complex constraints. A temperature like parameter, $\mathrm{T}$, is defined and gradually reduced in the optimisation process of SA. At each temperature, an iterative procedure, proposed by Metropolis et al [21] is performed.

A trail solution is obtained by perturbing the current solution according to a Gaussian probabilistic distribution function. If the cost of the trial solution is lower than that of the current solution, then it is accepted and used to generate another trial solution; else, the solution is accepted only when its transition probability of acceptance $P(T)$, given by Boltzmann distribution, is greater than a randomly generated number between 0 and 1 .

$P(T)=e^{\frac{-\Delta F}{T_{t}}}$

At each temperature, the procedure for generating and testing the trial solutions are repeated for an appropriate period of time (i.e. for an appropriate number of iterations) in order to allow the algorithm to settle into its thermal equilibrium i.e. a balanced state. If this time is too short, the algorithm is likely to converge to a local minimum. The combination of temperature steps and cooling times is known as the annealing schedule, which is usually selected empirically. The temperature is then reduced by the following geometric function

$T_{t+1}=\alpha \cdot T_{t}$

and the above mentioned iterative process is repeated till there is no significant improvement in the solution after a prespecified number of iterations. It can also be terminated when the maximum number of iterations is reached. It is to be noted that accepting deteriorated solutions in the above process enables the SA solutions to jump out of the local optimum solution points and paves the way to seek global optimum solutions.

\subsection{Proposed Methodology}

In all the existing SA based approaches for ELD, the real power generation of all generating units are considered as the decision variables that makes the size of the problem vary large, slow down the speed of these algorithms and hence not suitable for systems having larger number of generating units. In the proposed approach, the $\lambda$ of the classical $\lambda$ - iteration approach is considered as the only decision variable irrespective of the number of generating units. The real power generation of all the generating plants are considered as the problem dependant variables and expressed as a function of $\lambda$. In this approach, the real power generations are computed using Eq. (6) for each $\lambda$ value obtained during the SA iterations.

In the existing SA based ELD, the ranges for decision variables are the implicit lower and upper limits on real power generation of all the plants. But in the proposed approach, the lower and upper limits of the decision variable- $\lambda$ depend on the minimum and maximum power demands that the system can supply. The first step in obtaining these values is to compute the lower and upper incremental cost values by substituting the respective generation power limits in Eq. (6) for all the plants as

$$
\begin{aligned}
& I C_{i}{ }^{\min }=2\left[a_{i}+h_{i} d_{i}\right] P_{G i}^{\min }+\left[b_{i}+h_{i} e_{i}\right] \\
& I C_{i}{ }^{\max }=2\left[a_{i}+h_{i} d_{i}\right] P_{G i}^{\max }+\left[b_{i}+h_{i} e_{i}\right]
\end{aligned} \quad i=1,2, \cdots, n g
$$

The next step is choosing the lowest and highest incremental cost values, obtained from Eq. (9), as the limits for $\lambda$.

$$
\begin{aligned}
& \lambda^{\min }=\operatorname{Min}\left(I C_{1}^{\min }, I C_{2}^{\min }, \cdots, I C_{n g}^{\min }\right) \\
& \lambda^{\max }=\operatorname{Max}\left(I C_{1}^{\max }, I C_{2}^{\max }, \cdots, I C_{n g}^{\max }\right)
\end{aligned}
$$

The SA searches for the optimal solution by minimising a cost function. In the proposed formulation, the net fuel cost all the generating plants is considered as the cost function. However, a penalty term is included in the cost function to handle the explicit power balance constraint. The penalty term increases the cost of the function for infeasible 
solutions. The cost function is therefore built as a blend of fuel cost function and the power balance constraint through the use of a penalty factor as

Minimise $\operatorname{COST}=\sum_{i=1}^{n g} F_{i}\left(P_{G i}\right)+\lambda\left[\sum_{i=1}^{n g} P_{G i}-P_{D}\right]^{2}$

The number of decision variables in this formulation is always one, whereas the existing SA based approaches require the generation of all the plants as the variables. This reduction in decision variables will reduce the overall computational burden and improves the convergence rate. The algorithm of the proposed solution methodology for solving the ELD problem is outlined.

1. Read the input data of the EED problem

2. Set $i=1$

3. Choose initial temperature $T_{t}$, cooling coefficient $\alpha$, number of iterations for each temperature $N_{t}$ and maximum number of iterations $N_{\max }$.

4. Choose a random start point $\lambda_{o}$ in the specified range

5. Repeat the following till $i \% N_{t}=0$

a. Select a random point $\lambda_{i}$ from the neighbourhood of $\lambda_{o}$ within the specified range.

b. Solve Eq. (6) for $P_{G i}$ while imposing the limits given by Eq. (3).

c. Calculate $\operatorname{COST}_{i}$ using Eq. (11)

d. If $C O S T_{i}<C O S T_{o}$ then accept the trial solution by setting $\lambda_{o}=\lambda_{i}$

Else select a random number $\mathfrak{R}$ in the range $[0,1]$

$$
\text { if } P(T)>\Re \text {, then } \lambda_{o}=\lambda_{i} \text {, otherwise discard the trial point }
$$

e. Check for convergence by comparing the number of iterations $i$ with $N_{\text {max }}$. If converged, stop and print the ELD corresponding to the $\lambda_{o}$. Otherwise, set $i=i+1$

6. Reduce the temperature by the factor $\alpha$ using Eq. (12) and go to step (5).

\subsection{Simulation Results}

The proposed algorithm is tested on two examples, a six and an eleven generator systems, the data of which are available in Tables 1 and 2 respectively. The simulation is performed over a wide range of load demand using Matlab tool box and the results of PA are compared with that of classical $\lambda-$ iteration method, the methods suggested in References [11] and [13], and existing SA (ESA) strategy.

The generator allocations for the different load demands for both systems are tabulated in Tables 3 and 4 respectively. The fuel cost for both test systems are compared in Tables 5 and 6 respectively. It is observed that the PA allows to incur the same cost as that of the traditional $\lambda-$ iteration method, thus validating the performance of the new approach. The fuel cost for the other two methods cited in ref [11] and [13] also closely fall in line. It is only in the ESA, where the cost accrues to be slightly higher.

The emissions for the examples under study are compared in Tables 7 and 8 respectively. It is worthy to note that the emissions are exactly as that of $\lambda-$ iteration method thus once again illustrating the merits of the new strategy. The emissions of the remaining two are also similar except that in ESA, where it is slightly lower. This is due to the fact that the increased fuel cost in ESA is augmented by a considerably smaller emission. 
It is highly satisfying to note from Table 9 that the normalised execution time (NET) of PA is very low when compared to ESA and it is almost constant to both the systems, thus emphasising the fact that the performance of the new algorithm is independent of system size. It has to be acknowledged that in the case of ESA, the NET is much larger and almost increases exponentially with the system size.

Table 1 Data for 6 Generator system

\begin{tabular}{|c|c|c|c|c|c|c|c|c|}
\hline \multirow{2}{*}{$\begin{array}{c}\text { Gen } \\
\text { No }\end{array}$} & \multicolumn{3}{|c|}{ Fuel cost coefficients } & \multicolumn{3}{c|}{ Emission coefficients } & \multicolumn{2}{c|}{ Generation limits } \\
\cline { 2 - 9 } & $a$ & $b$ & $c$ & $d$ & $e$ & $f$ & $P_{G}{ }^{\min }$ & $P_{G}{ }^{\max }$ \\
\hline 1 & 0.1525 & 38.540 & 756.800 & 0.00420 & 0.3300 & 13.860 & 10 & 125 \\
\hline 2 & 0.1060 & 46.160 & 451.325 & 0.00420 & 0.3300 & 13.860 & 10 & 150 \\
\hline 3 & 0.0280 & 40.400 & 1050.00 & 0.00683 & -0.5455 & 40.267 & 35 & 225 \\
\hline 4 & 0.0355 & 38.310 & 1243.53 & 0.00683 & -0.5455 & 40.267 & 35 & 210 \\
\hline 5 & 0.0211 & 36.328 & 1658.57 & 0.00460 & -0.5112 & 42.900 & 130 & 325 \\
\hline 6 & 0.0180 & 38.270 & 1356.66 & 0.00460 & -0.5112 & 42.900 & 125 & 315 \\
\hline
\end{tabular}

Table 2 Data for 11 Generator system

\begin{tabular}{|c|c|c|c|c|c|c|c|c|}
\hline \multirow{2}{*}{$\begin{array}{c}\text { Gen } \\
\text { No }\end{array}$} & \multicolumn{3}{|c|}{ Fuel cost coefficients } & \multicolumn{3}{c|}{ Emission coefficients } & \multicolumn{2}{c|}{ Generation limits } \\
\cline { 2 - 9 } & $a$ & $b$ & $c$ & $d$ & $e$ & $f$ & $P_{G}^{\min }$ & $P_{G}^{\max }$ \\
\hline 1 & 0.00762 & 1.92699 & 387.85 & 0.00419 & -0.67767 & 33.93 & 20 & 250 \\
\hline 2 & 0.00838 & 2.11969 & 441.62 & 0.00461 & -0.69044 & 24.62 & 20 & 210 \\
\hline 3 & 0.00523 & 2.19196 & 422.57 & 0.00419 & -0.67767 & 33.93 & 20 & 250 \\
\hline 4 & 0.00140 & 2.01983 & 552.50 & 0.00683 & -0.54551 & 27.14 & 60 & 300 \\
\hline 5 & 0.00154 & 2.22181 & 557.75 & 0.00751 & -0.40060 & 24.15 & 20 & 210 \\
\hline 6 & 0.00177 & 1.91528 & 562.18 & 0.00683 & -0.54551 & 27.14 & 60 & 300 \\
\hline 7 & 0.00195 & 2.10681 & 568.39 & 0.00751 & -0.40006 & 24.15 & 20 & 215 \\
\hline 8 & 0.00106 & 1.99138 & 682.93 & 0.00355 & -0.51116 & 30.45 & 100 & 455 \\
\hline 9 & 0.00117 & 1.99802 & 741.22 & 0.00417 & -0.56228 & 25.59 & 100 & 455 \\
\hline 10 & 0.00089 & 2.12352 & 617.83 & 0.00355 & -0.41116 & 30.45 & 110 & 460 \\
\hline 11 & 0.00098 & 2.10487 & 674.61 & 0.00417 & -0.56228 & 25.59 & 110 & 465 \\
\hline
\end{tabular}


Table 3 Generation allocations for 6-generator system in MW

\begin{tabular}{|c|c|c|c|c|c|c|}
\hline$P_{D}$ & $P_{G 1}$ & $P_{G 2}$ & $P_{G 3}$ & $P_{G 4}$ & $P_{G 5}$ & $P_{G 6}$ \\
\hline 500 & 19.999 & 14.899 & 93.051 & 90.064 & 143.647 & 138.339 \\
\hline 600 & 31.723 & 28.662 & 108.405 & 103.960 & 166.507 & 160.744 \\
\hline 700 & 43.446 & 42.426 & 123.758 & 117.855 & 189.366 & 183.148 \\
\hline 800 & 55.170 & 56.189 & 139.112 & 131.751 & 212.225 & 205.552 \\
\hline 900 & 66.894 & 69.953 & 154.466 & 145.647 & 235.084 & 227.957 \\
\hline 1000 & 78.617 & 83.716 & 169.820 & 159.542 & 257.943 & 250.361 \\
\hline 1100 & 90.341 & 97.479 & 185.174 & 173.438 & 280.802 & 272.765 \\
\hline
\end{tabular}

Table 4 Generation allocations for 11-generator system in MW

\begin{tabular}{|c|c|c|c|c|c|c|c|c|c|c|c|}
\hline$P_{D}$ & $P_{G 1}$ & $P_{G 2}$ & $P_{G 3}$ & $P_{G 4}$ & $P_{G 5}$ & $P_{G 6}$ & $P_{G 7}$ & $P_{G 8}$ & $P_{G 9}$ & $P_{G 10}$ & $P_{G 11}$ \\
\hline 1000 & 85.61 & 76.67 & 87.26 & 78.50 & 47.92 & 79.33 & 49.77 & 129.60 & 122.37 & 119.60 & 123.40 \\
\hline 1250 & 94.62 & 82.69 & 97.02 & 102.33 & 62.73 & 102.53 & 64.85 & 165.51 & 156.89 & 160.27 & 160.56 \\
\hline 1500 & 103.63 & 88.71 & 106.77 & 126.17 & 77.54 & 125.73 & 79.93 & 201.42 & 191.41 & 200.95 & 197.74 \\
\hline 1750 & 112.64 & 94.73 & 116.53 & 150.01 & 92.35 & 148.91 & 95.01 & 237.33 & 225.93 & 241.62 & 234.91 \\
\hline 2000 & 121.65 & 100.75 & 126.29 & 173.85 & 107.15 & 172.16 & 110.10 & 273.23 & 260.45 & 282.30 & 272.08 \\
\hline 2250 & 130.66 & 106.76 & 136.05 & 197.69 & 121.96 & 195.37 & 125.18 & 309.14 & 294.97 & 322.97 & 309.26 \\
\hline 2500 & 139.67 & 112.78 & 145.80 & 221.53 & 136.77 & 218.58 & 140.26 & 345.05 & 329.48 & 363.65 & 346.43 \\
\hline
\end{tabular}

Table 5 Comparison of fuel cost in $\$ / h$ for 6 -generator system

\begin{tabular}{|c|c|c|c|c|c|}
\hline$P_{D}$ & $\lambda-$ iteration & Ref [11] & Ref [13] & ESA & PA \\
\hline 500 & 27092.42 & 27092.46 & 27092.46 & 27096.47 & 27092.42 \\
\hline 600 & 31628.63 & 31628.64 & 31628.63 & 31629.99 & 31628.63 \\
\hline 700 & 36313.92 & 36313.94 & 36313.92 & 36315.75 & 36313.92 \\
\hline 800 & 41148.31 & 41148.33 & 41148.32 & 41152.50 & 41148.31 \\
\hline 900 & 46131.86 & 46131.85 & 46131.87 & 46133.67 & 46131.86 \\
\hline 1000 & 51264.41 & 51264.49 & 51264.47 & 51266.42 & 51264.41 \\
\hline 1100 & 56546.15 & 56546.18 & 56546.17 & 56561.31 & 56546.15 \\
\hline
\end{tabular}

Table 6 Comparison of fuel cost in $\$ / h$ for 11 -generator system

\begin{tabular}{|c|c|c|c|c|c|}
\hline$P_{D}$ & $\lambda-$ iteration & $\operatorname{Ref}[11]$ & $\operatorname{Ref}[13]$ & ESA & PA \\
\hline 1000 & 8502.30 & 8502.29 & 8502.29 & 8502.71 & 8502.30 \\
\hline 1250 & 9108.38 & 9108.38 & 9108.38 & 9108.78 & 9108.38 \\
\hline 1500 & 9733.53 & 9733.54 & 9733.54 & 9733.93 & 9733.53 \\
\hline 1750 & 10377.78 & 10377.77 & 10377.77 & 10379.47 & 10377.78 \\
\hline 2000 & 11041.09 & 11041.08 & 11041.08 & 11043.65 & 11041.09 \\
\hline 2250 & 11723.47 & 11723.47 & 11723.47 & 11724.10 & 11723.47 \\
\hline 2500 & 12424.94 & 12424.94 & 12424.94 & 12426.01 & 12424.94 \\
\hline
\end{tabular}


Table 7 Comparison of emission in $R s / k g$ for 6-generator system

\begin{tabular}{|c|c|c|c|c|c|}
\hline$P_{D}$ & $\lambda-$ iteration & $\operatorname{Ref}[11]$ & $\operatorname{Ref}[13]$ & ESA & PA \\
\hline 500 & 261.63 & 261.63 & 261.63 & 261.39 & 261.63 \\
\hline 600 & 338.99 & 338.99 & 338.99 & 338.05 & 338.99 \\
\hline 700 & 434.38 & 434.38 & 434.38 & 434.17 & 434.38 \\
\hline 800 & 547.79 & 547.80 & 547.80 & 547.63 & 547.79 \\
\hline 900 & 679.24 & 679.24 & 679.24 & 678.58 & 679.24 \\
\hline 1000 & 828.71 & 828.72 & 828.72 & 828.14 & 828.71 \\
\hline 1100 & 996.22 & 996.22 & 996.22 & 995.62 & 996.22 \\
\hline
\end{tabular}

Table 8 Comparison of emission in $R s / k g$ for 11-generator system

\begin{tabular}{|c|c|c|c|c|c|}
\hline$P_{D}$ & $\lambda-$ iteration & $\operatorname{Ref}[11]$ & $\operatorname{Ref}[13]$ & ESA & PA \\
\hline 1000 & 205.20 & 205.20 & 205.20 & 205.18 & 205.20 \\
\hline 1250 & 339.87 & 339.87 & 339.87 & 339.63 & 339.87 \\
\hline 1500 & 540.54 & 540.54 & 540.54 & 540.60 & 540.54 \\
\hline 1750 & 807.23 & 807.22 & 807.22 & 806.09 & 807.23 \\
\hline 2000 & 1139.91 & 1139.91 & 1139.91 & 1138.62 & 1139.91 \\
\hline 2250 & 1538.60 & 1538.60 & 1538.60 & 1536.23 & 1538.60 \\
\hline 2500 & 2003.30 & 2003.30 & 2003.30 & 2002.15 & 2003.30 \\
\hline
\end{tabular}

Table 9 Normalized Execution Time in seconds

\begin{tabular}{|c|c|c|}
\hline Test System & ESA & PA \\
\hline 6 Generator System & 482 & 23.50 \\
\hline 11 Generator System & 1030 & 28.45 \\
\hline
\end{tabular}

\subsection{CONCLUSION}

A new strategy involving SA for solving EED problem has been developed with a view to lower the computational burden and renders it suitable for online applications. The close agreement of the performance of PA with the benchmark $\lambda-$ iteration method, besides the other two formulations obtained from references [11] and [13] has allowed to acclaim its accuracy. The fact that it has been coined with a single decision variable facilitates it with a smaller search horizon and cool down at the global optimal solution vary fast. It is implicit that the new approach fosters the continued use of SA and will go a long way in serving as a useful tool in load dispatch centres.

\section{Acknowledgement}

The authors gratefully acknowledge the authorities of Annamalai University for the facilities offered to carry out this work.

\section{REFERENCE}

1. Wood. AJ \& Woolenberg. BF. (1996). Power generation, operation and control, John Willey \& Sons, New York.

2. Chowdhury.B.H and Rahman. S. (1990). A review of recent advances in economic dispatch, IEEE Trans. on Power Systems, 5(4), 1248-1259.
3. Al-Sumait. J.S, Sykulski. J.K, Al-Othman. A.K. (2008). Solution of different types of economic load dispatch problems using a pattern search method, Electric Power Components and Systems, 36, 250265.

4. Abdelaziz. A.Y, Mekhamer. S.F, Badr. M.A.L and Kamh. M.Z. (2008). Economic Dispatch using an enhanced Hopfield neural network, Electric Power Components and Systems, 36, 719-732.

5. Mekhamer. S.F, Abdelaziz. A.Y, Kamh. M.Z and Badr. M.A.L. (2009). Dynamic economic dispatch using a hybrid neural network quadratic programming based technique, Electric Power Components and Systems, 37, 253-264.

6. Adhinarayanan. T and Sydulu. M. (2008). Diretional search genetic algorithm applications to economic dispatch of thermal units, International Journal for Computational Methods in Engineering Science and Mechanics, 9, 211-216.

7. Panigrahi. C.K, Chattopadhyah. P.K, Chakrabarti. R.N and Basu. N. (2006). Simulated annealing technique for dynamic economic dispatch, Electric Power Components and Systems, 34, 577-586. 
8. Manoharan. P.S, Kannan. P.S, Baskar. S, Willjuice Iruthayarajan and Dhananjeyan. (2009). Covarience matrix adopted evolution strategy algorithm-based solution to dynamic economic dispatch problems, Engineering Optimization, 41(7), 635-657.

9. Immanuel Selvakumar. A and Thanuskodi. K. (2008). Anti-predatory particle swarm optimisation: solution to nonconvex economic dispatch problems, Electric Power Systems Research, 78, 2-10.

10. Shin-Der Chan and Jiann-Fuh Chen. (2003). A direct Newton-Raphson economic emission dispatch, Electrical Power and Energy Systems, 25, 411-17.

11. Muralidharan. S, Srikrishna. K and Subramanian. (2006). Emission constrained economic dispatch-a new recursive approach, Electric Power Components and Systems, 34, 343-53.

12. Tankut. Y and Onur. K. (2007). A multiobjective optimisation method to environmental economic dispatch, Electrical Power and Energy Systems, 29, 42-50.

13. Balamurugan. R and Subramanian. S. (2008). A simplified recursive approach to combined economic emission dispatch, Electric Power Components and Systems, 36(1), 17-27.

14. Palanichamy. C and Sundar Babu. (2008). Analytical solution for combined economic and emission dispatch, Electric Power Systems Research, 78, 112937.
15. Basu. M. (2004). An interactive fuzzy satisfying-based simulated annealing technique for economic emission load dispatch with nonsmooth fuel cost and emission level functions, Electric Power Components and Systems, 36, 163-73.

16. Basu. M. (2006). Particle swarm optimization based goal-attainment method for dynamic economic emission dispatch, Electric Power Components and Systems, 34, 1015-25.

17. Lingfeng Wang and hanan Singh. (2007). Environmental/economic power dispatch using a fuzzified multi-objective particle swarm optimisation algorithm, Electric Power Systems Research, 77, 1654-64.

18. Osman. M.S, Abo-Sinna. M.A and Mousa. A.A. (2009). An $\mathcal{E}$-dominance-based multiobjective genetic algorithm for economic emission load dispatch optimisation problem, Electric Power Systems Research, 79(11), pp. 1561-67.

19. Palanichamy. C and Srikrishna. K. (1991). Economic thermal power dispatch with emission constraint, J. Indian Institute of Eng. (India), 72, pp. 11-18.

20. Kirkpatrick. S, Gelatt Jr. C.D and Vecchi. M.P (1983). Optimization by simulated annealing, Science, 220, 671-80.

21. Metropolis. N, Rosenbluth. A.W, Rosenbluth. M.N, Teller. A.H and Teller. E. (1953). Equations of state calculations by fast computing machines, J Chem Phys, 21, 1087-92. 